\title{
Small-scale flux emergence events observed by Sunrise/IMaX
}

\section{S. L. Guglielmino ${ }^{1,2}$, V. Martínez Pillet ${ }^{1}$, J. C. del Toro Iniesta ${ }^{3}$, L. R. Bellot Rubio ${ }^{3}$, F. Zuccarello ${ }^{4}$, S. K. Solanki ${ }^{5,6}$ \& the Sunrise/IMaX team}

\author{
${ }^{1}$ IAC Instituto de Astrofísica de Canarias, \\ C/ Vía Láctea s/n, La Laguna, Tenerife, E-38200, Spain \\ email: sgu@iac.es \\ ${ }^{2}$ ULL Departamento de Astrofísica, Univ. de La Laguna, \\ La Laguna, Tenerife, E-38205, Spain \\ ${ }^{3}$ IAA Instituto de Astrofísica de Andalucía (CSIC), \\ Apdo. 3004, Granada, E-18080, Spain \\ ${ }^{4}$ Dipartimento di Fisica e Astronomia - Sezione Astrofisica, \\ Via S. Sofia 78, 95123 Catania, Italy \\ ${ }^{5}$ Max-Planck-Institut für Sonnensystemforschung, \\ Max-Planck-Str. 2, 37191 Katlenburg-Lindau, Germany \\ ${ }^{6}$ School of Space Research, Kyung Hee University, \\ Yongin, Gyeonggi 446-701, Republic of Korea
}

\begin{abstract}
Thanks to the unprecedented combination of high spatial resolution $\left(0^{\prime \prime} .2\right)$ and high temporal cadence (33 s) spectropolarimetric measurements, the IMaX magnetograph aboard the Sunrise balloon-borne telescope is revealing new insights about the plasma dynamics of the all-pervasive small-scale flux concentrations in the quiet Sun. We present the result of a case study concerning the appearance of a bipole, with a size of about $4^{\prime \prime}$ and a flux content of $5 \times 10^{17}$ $\mathrm{Mx}$, with strong signal of horizontal fields during the emergence. We analyze the data set using the SIR inversion code and obtain indications about the three-dimensional shape of the bipole and its evolution with time.
\end{abstract}

Keywords. Sun: photosphere, Sun: magnetic fields, Sun: activity, Techniques: high angular resolution

\section{IMaX Observations and Data Analysis}

A small-scale emerging bipole was observed in 09 June 2009 near the disk center at high spatial resolution with the Imaging Magnetograph eXperiment (IMaX, Martínez Pillet et al., 2010), mounted on the 1-m aperture telescope flown during the Sunrise mission, a balloon-borne solar observatory (Barthol et al., 2010; Solanki et al., 2010). IMaX took polarization maps at five wavelength positions over the Fe I $525.02 \mathrm{~nm}$ line, at -80, -40, $+40,+80,+227 \mathrm{m \AA}$ from the line center, with a cadence of $33 \mathrm{~s}$ and a pixel size of $0^{\prime \prime} .055$, recording the full Stokes parameters $I, Q, U$, and $V$ over the full IMaX field-ofview $(\mathrm{FOV})$ of about $50^{\prime \prime} \times 50^{\prime \prime}$. In this study we analyze non-reconstructed data, as the Phase-Diversity technique used to obtain reconstructed data implies an apodization of the full FOV of IMaX, and the emerging bipole appeared just at its right border. 


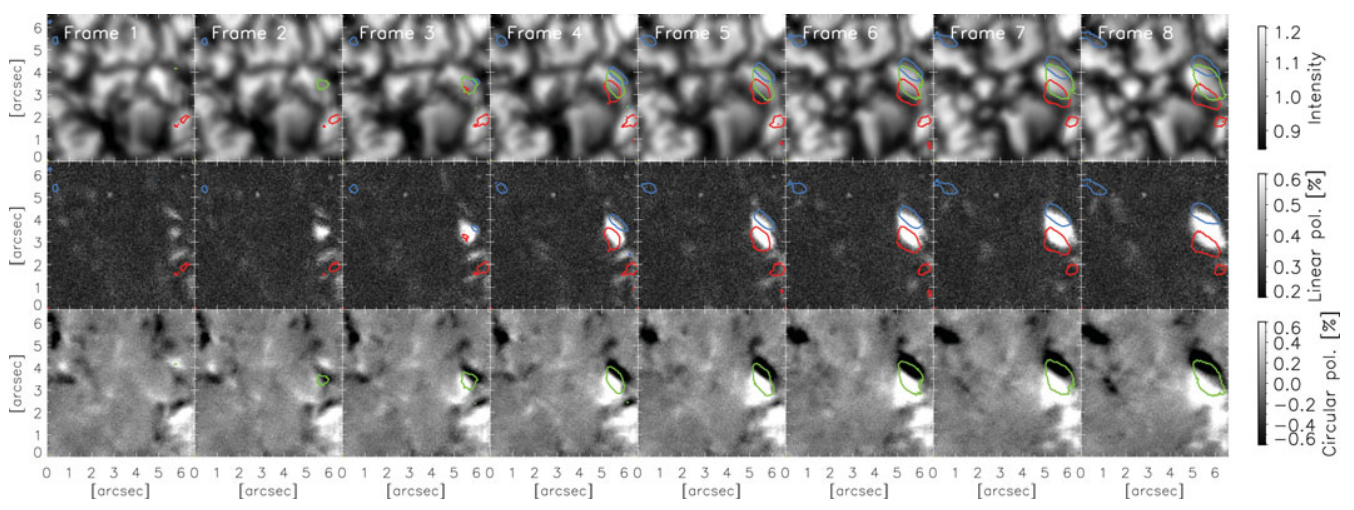

Figure 1. Top row: Time evolution of the continuum maps during the appearance of the bipole; red (blue) contours represent a circular polarization signal of $+0.8(-0.8) \%$ of the $I_{c}$; green contours over the circular polarization maps represent a linear polarization signal of $0.5 \%$ of the $I_{c}$. Middle row: the same for the mean linear polarization signal $L_{s}$. Bottom row: the same for the mean circular polarization signal $V_{s}$. The bipole appears in the frame 1, giving rise to a considerable signal in the $L_{s}$ map. In the frame 3 we see two small patches of opposite polarity at opposite edges of the structure seen in the $L_{s}$ map. The evolution of the emerging loop is clearly recognizable in the subsequent frames, where opposite polarities of the bipole separate each other in opposite directions, with a quite strong $L_{s}$ signal in between them, which coincides with the top of a granule in the continuum map.

We obtained maps of the mean circular polarization signal $V_{s}$ and of the mean linear polarization signal $L_{s}$, given by

$$
V_{s}=1 / 4\left\langle I_{c}\right\rangle \epsilon \sum_{i=1}^{4}\left|V_{i}\right| \text { and } L_{s}=1 / 4\left\langle I_{c}\right\rangle \sum_{i=1}^{4} \sqrt{{Q_{i}}^{2}+U_{i}{ }^{2}},
$$

where $I_{c}$ is the continuum intensity averaged over the IMaX FOV, $\epsilon= \pm 1$ depends on the sign of the blue lobe of Stokes $V$, and $i$ runs over the first four wavelength positions. The signal-to-noise ratio in these measurements is about $2 \cdot 10^{3}$.

We carried out inversions of the observed Stokes vector using the SIR code (Ruiz Cobo \& del Toro Iniesta, 1992), which yields the temperature stratification, the line-ofsight (LOS) velocity, the magnetic field strength, inclination and azimuth angles in the LOS reference frame. While these latter are assumed by SIR inversion to be constant throughout the photosphere, the LOS velocity and magnetic field strength have a linear gradient. Such a gradient has been found to be necessary in order to fit some profiles that show a considerable asymmetry between the blue and red lobes in Stokes $V$. No stray light contamination was taken into account for the inversions.

\section{Results}

In Figure 1 we display the temporal sequences of the mean linear polarization and mean circular polarization maps, that clearly show the emergence and the evolution of the bipole at the beginning of its appearance: first a patch with a strong linear polarization signal appears, and a pair of structures with opposite polarities is seen at the opposite edges of the patch in the following frame. Then the opposite polarities separate in time. In the continuum map is clearly visible that the polarization signals coincide with a granule. 
Summarizing the results obtained from the SIR inversion:

- upflow motions are found in correspondence of the region with strong linear polarization signal;

- magnetic field strength and flux grow in time, reaching a peak about five minutes since the first detection of the bipole;

- footpoints do not reach a vertical orientation, but rather they remain inclined at about $45^{\circ}$ with respect to the vertical;

- in the emergence zone, the azimuth angle is quite homogeneous, ranging from $25^{\circ}$ to $55^{\circ}$;

- the magnetic flux content of the bipole at its maximum is of about $5 \times 10^{17} \mathrm{Mx}$, while the size of about $4^{\prime \prime}$, roughly of the same order of a granule.

We have also produced the scatter plots of the magnetic field strength and LOS velocity vs. zenith angle. The footpoints of the loop have a field strength of about $300 \mathrm{G}$ and downflows up to $3 \mathrm{~km} \mathrm{~s}^{-1}$, while the horizontal parts show upflows of $1.5 \mathrm{~km} \mathrm{~s}^{-1}$, decreasing with time. In both cases, no evidence of asymmetry between the footpoints of the bipole is found in the data.

\section{Conclusions}

IMaX/Sunrise observations of the solar photosphere taken at the disk center have revealed a number of small-scale episodes of magnetic flux emergence (Danilovic et al., 2010; Solanki et al., 2010). We have carried out an analysis concerning the emergence of a small magnetic bipole. We have analyzed the polarization maps and then we have inverted the Stokes profiles with the SIR code, to obtain information on the physical parameters of this magnetic structure.

The magnetic flux content locates this small bipole in a halfway point between the ephemeral regions studied by Hagenaar (2001) and the all-pervasive loops of the quiet Sun. The bipole indeed appears to be coincident with a granule in the continuum map, so it would apparently represent a typical case of flux emergence at granular scale. Nevertheless, the footpoints separate up to $4500 \mathrm{~km}$ and their remnants remain visible until 15 minutes since the first detection of the magnetic structure. There are also evidences for opposite motions along the LOS at different altitude in the atmosphere in the same pixels: upflows in the higher layers and downflows in the deeper layers at the external border of the negative footpoint might indicate the presence of small-scale jets, due to the interaction of the emerging loop with the ambient magnetic field.

\section{Acknowledgements}

This work has been partially funded by the Spanish Ministerio de Educación y Ciencia, through Projects ESP2006-13030-C06-01/02/03/04 and AYA2009-14105-C06-03/06 INTA+GACE, and Junta de Andalucía, through Project P07-TEP-2687, including a percentage from European FEDER funds. Financial support by the European Commission through the SOLAIRE Network (MTRN-CT-2006-035484) is gratefully acknowledged.

\section{References}

Barthol, P., et al. 2010, Solar Phys., in press

Danilovic, S., et al. 2010, Astrophys. Jour. Lett., 723, L149

Hagenaar, H. J., 2001, Astrophys. Jour., 555, 448

Martnez Pillet, V., et al. 2010, Solar Phys., in press

Ruiz Cobo, B. \& del Toro Iniesta, J.C. 1992, Astrophys. Jour., 398, 375

Solanki, S. K., et al. 2010, Astrophys. Jour. Lett., 723, L127 